\title{
Channel Equalisation of a MIMO FBMC/OQAM System Using a Polynomial Matrix Pseudo-Inverse
}

\author{
Amr A. Nagy and Stephan Weiss \\ Dept. of Electronic and Electrical Engineering, University of Strathclyde, Glasgow G1 1XW, Scotland \\ Email: amr.a.nagy,stephan.weiss@strath.ac.uk
}

\begin{abstract}
When using filter bank based multicarrier orthogonal quadrature amplitude modulation (FBMC/OQAM) techniques in a multiple-input multiple-output (MIMO) environment, its difficulty of dealing with inter-symbol interference (ISI) and inter-carrier interference (ICI) is further exacerbated by the presence of spatial interference. In this paper, we describe the transfer functions (including all temporal and spatial interference terms) by polynomial matrices. The equalisation of this system can then be performed by a proposed polynomial matrix pseudo-inverse. Some numerical examples for this approach are presented.
\end{abstract}

\section{INTRODUCTION}

Filter bank based multicarrier (FBMC) modulation methods are a strong candidate for 5 th generation communications systems and beyond because of their robustness to synchronisation errors when compared to orthogonal frequency division multiplexing (OFDM) systems [5]. Different from OFDM, FBMC creates subcarriers that retain at least some frequencyselectivity. Even though recent practical measurements indicate that for a well-synchronised FBMC system, single tap equalisers may be sufficient [14], generally significant dispersion has to be considered [3], [12], if not through long channel impulse response then through insufficient timing synchronisation of the receiver [13]. As a result, significant inter-symbol interference (ISI) arises.

Amongst the different FBMC choices, a critically sampled DFT filter bank combined with judicious interleaving of real- and imaginary parts akin to offset-quadrature amplitude modulation (OQAM) has attracted attention as it reaches maximum spectrum efficient yet retains orthogonality at least for the real part of the transmitted symbols [18]. Due to critical sampling, at least adjacent subbands overlap spectrally, causing inter-carrier interference (ICI) in the receiver. Thus equalisers cannot operate per-band but need to incorporate cross-terms with at least adjacent bands akin early subband filtering schemes such as [6]. Nevertheless, the high spectral efficiency of FBMC/OQAM has motivated numerous solutions that aim to solve this problem [13]-[15].

When considering FBMC/OQAM for transmission over MIMO channels, the interference situation is worsened by additional inter-antenna interference (IAI) terms in addition to ISI and ICI. This is generally seen as a significant problem, and substantial efforts have been undertaken to combat these interference terms - see e.g. early works in [7], or more recently in [3], [12] or the review in [15]. The majority of these publications has targetted the joint design of precoders and equalisers, which can enable significant benefits if the channel is know to both receiver and transmitter.

In the absence of channel state information at the transmitter, often efforts have to be restricted to equalisation, and the aim of this paper is propose such an approach. Our design is based on the modelling of the inner part of the FBMC/OQAM system - including the filter banks and the MIMO channel, which are responsible for dispersion and synchronisation errors - by an equivalent polynomial channel matrix. We then use algebraic techniques for such matrices to find an equaliser implementation. The polynomial notation and problem formulation of a broadband communication systems is not news - see e.g. [11] for a SISO and [12] for a MIMO precoder and equaliser - but solutions to such problems have not been straightforward. We here rely in novel polynomial matrix formulations [21] and associated algorithms [4], [9], [16], [17] to calculate a polynomial matrix pseudo-inverse as equaliser as a MIMO extension of the work in [13]. The advantage of these polynomial matrix techniques lie in the coherent treatment of the problem, which avoids the challenge of association of bin-wise solution from DFT bin to DFT bin [12].

This paper is organised as follows: Sec. II reviews the FBMC/OQAM system model, which motivates the description of the MIMO channel combined with the synthesis and analysis filter banks of the FBMC/OQAM system by an equivalent channel matrix in Sec. III. The equalisation of the MIMO-FBMC/OQAM system via the pseudo-inversion of this equivalent channel matrix is the topic of Sec. IV, followed by a numerical example and conclusions in Secs. V and VI, respectively.

\section{SySTEM MOdEL}

An FBMC/OQAM system for a MIMO channel with $N_{\mathrm{T}}$ transmitters and $N_{\mathrm{R}}$ receivers is outlined in Fig. 1. For a brief description of FBMC/OQAM, with reference to Fig. 1 we first assume the single input single output case with $N_{\mathrm{T}}=$ $N_{\mathrm{R}}=K=1$, and $\mathbf{W}[n] \circ-\bullet \boldsymbol{W}(z)=\mathbf{I}_{M}$. In the inner, shaded part of the FBMC system in Fig. 1, a DFT-modulated filter bank with a synthesis filter bank (SFB) and an analysis filter bank (ASB) multiplexes a signal vector $\mathbf{u}[n] \in \mathbb{C}^{M}$ across the channel, employing an upsampling ratio of $M / 2$. In the receiver, the signal is demultiplexed into $\mathbf{x}[n] \in \mathbb{C}^{M}$. For a DFT filter bank to permit maximum symbol density (and hence maximum spectral efficiency) while satisfying 


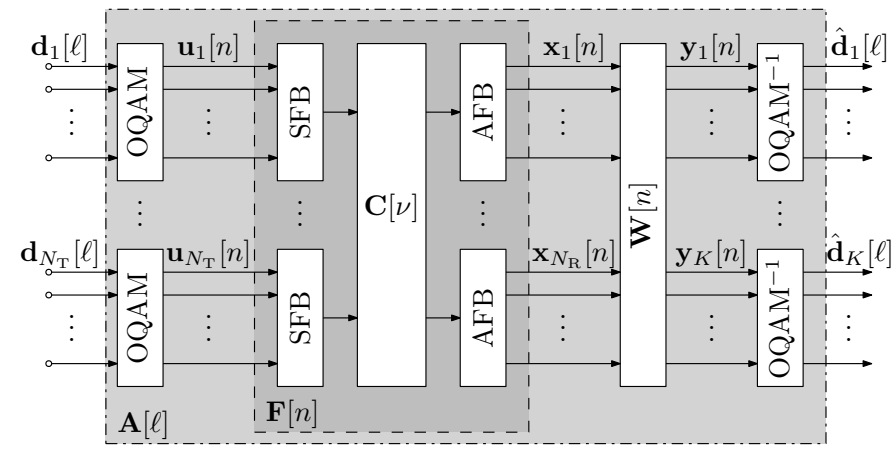

Fig. 1. FBMC/OQAM system transmultiplexing signals over a MIMO channel $\mathbf{C}[n]$ with $N_{\mathrm{T}}$ transmitters and $N_{\mathrm{R}}$ receivers with equaliser $\mathbf{W}[n]$.

good localisation in both time and frequency domains, the orthogonality of the system has to be relaxed.

In FBMC/OQAM the relaxation of conditions is achieved by restricting orthogonality to the real part only by judiciously assigning alternating real and imaginary values in $\mathbf{d}[\ell] \in \mathbb{C}^{M}$ to $\mathbf{u}[n] \in \mathbb{C}^{M}$ through a staggering and de-staggering process [18] akin to an OQAM modulation. As a result, the index $n$ runs twice as fast as the time index $\ell$. Through this, partial orthogonality is enforced, such that $\hat{\mathbf{d}}[\ell]=\mathbf{d}[\ell]$ if the channel is transparent.

For the MIMO case, we assume that $K$ spatial transmit channels can be formed, where $K=\min \left\{N_{\mathrm{T}}, N_{\mathrm{R}}\right\}$. An FBMC/OQAM block is applied to each of the transmitters and receivers, as shown in Fig. 1. An equaliser $\mathbf{W}[n] \in$ $\mathbb{C}^{M K \times M N_{\mathrm{R}}}$, applied prior to the OQAM de-staggering in the receiver, has the aim of equalising and synchronising the overall system. This is similar to the equaliser and synchroniser and [13], but here also aims to cancel cancel spatial or interantenna-interference.

\section{Equivalent Polynomial Channel Matrix}

In order to later define the equaliser $\boldsymbol{W}(z) \bullet-\circ \mathbf{W}[n]$, we first seek a description of the inner FBMC component of Fig. 1. For this, we utilise a polyphase notation of the analysis and synthesis filter banks. With $M$ channnels and an oversampling factor of 2 , the polyphase analysis matrix $\boldsymbol{H}(z): \mathbb{C} \rightarrow \mathbb{C}^{M \times M / 2}$ is an $M \times \frac{M}{2}$ polynomial matrix. Similarly, a synthesis filter bank can be described by a polyphase synthesis matrix $\boldsymbol{G}(z): \mathbb{C} \longrightarrow \mathbb{C}^{M / 2 \times M}$, where for a DFT filter bank, the polyphase synthesis matrix $\boldsymbol{G}(z)$ is the parahermitian of the polyphase analysis matrix, i.e. $\boldsymbol{G}(z)=\boldsymbol{H}^{\mathrm{P}}(z)=\boldsymbol{H}^{\mathrm{H}}\left(1 / z^{*}\right)$. To form filter banks, these polyphase realisations are combined with parallel-toserial $(\mathrm{p} / \mathrm{s})$ and serial-to-parallel ( $\mathrm{s} / \mathrm{p})$ converters, as depicted in Fig. 2.

If $C_{n_{\mathrm{R}}, n_{\mathrm{T}}}(z)$ is the channel transfer function between the $n_{\mathrm{T}}$ th transmitter, $n_{\mathrm{T}}=1 \ldots N_{\mathrm{T}}$, and the $n_{\mathrm{R}}$ th receiver, $n_{\mathrm{R}}=$ $1 \ldots N_{\mathrm{R}}$, the MIMO system transfer function between the $\mathrm{p} / \mathrm{s}$ input vector $\mathbf{s}_{n_{\mathrm{T}}}[n]$ and the $\mathrm{s} / \mathrm{p}$ output vector $\mathbf{r}_{n_{\mathrm{R}}}[n]$ is the

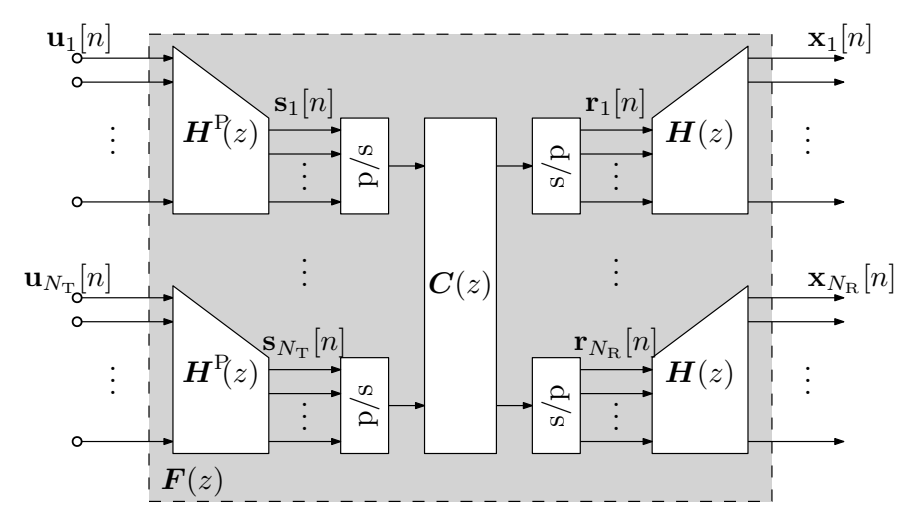

Fig. 2. Inner component of the MIMO FBMC/OQAM system in polyphase notation.

pseudo-circulant matrix $\boldsymbol{C}_{n_{\mathrm{R}}, n_{\mathrm{T}}}(z): \mathbb{C} \rightarrow \mathbb{C}^{M / 2 \times M / 2}[13]$, [16],

$\mathbf{C}_{n_{\mathrm{R}}, n_{\mathrm{T}}}(z)=\left[\begin{array}{cccc}C_{0}(z) & C_{1}(z) & \cdots & C_{\frac{M}{2}-1}(z) \\ z^{-1} C_{\frac{M}{2}-1}(z) & C_{0}(z) & \cdots & C_{\frac{M}{2}-2}(z) \\ \vdots & & \ddots & \vdots \\ z^{-1} C_{1}(z) & \cdots & z^{-1} C_{\frac{M}{2}-1}(z) & C_{0}(z)\end{array}\right]$.

For notational brevity, the subscripts $\{\cdot\}_{n_{R}, n_{\mathrm{T}}}$ have been omitted from the r.h.s. of the above equation, which comprises of the $\frac{M}{2}$ type-I polyphase components $C_{\mu}(z), \mu=1 \ldots \frac{M}{2}$ of $C_{n_{\mathrm{R}}, n_{\mathrm{T}}}(z)$ [19] such that

$$
C_{n_{\mathrm{R}}, n_{\mathrm{T}}}(z)=\sum_{\mu=0}^{\frac{M}{2}-1} C_{\mu}\left(z^{\frac{M}{2}}\right) z^{-\mu}
$$

describes the channel between the $n_{\mathrm{T}}$ th transmitter and $n_{\mathrm{R}}$ th receiver.

The overall MIMO transfer function between the concatenated filter bank inputs $\left[\mathbf{u}_{1}^{\mathrm{H}}[n] \ldots \mathbf{u}_{N_{T}}^{\mathrm{H}}[n]\right]^{\mathrm{H}}$ in the transmitter and the concatenated vector of filter bank outputs $\left[\mathbf{x}_{1}^{\mathrm{H}}[n] \ldots \mathbf{x}_{N_{\mathrm{R}}}^{\mathrm{H}}[n]\right]^{\mathrm{H}}$ in the receiver is described by the matrix $\mathbf{F}[n] \circ-\bullet \boldsymbol{F}(z)$, which forms the shaded system parts in Figs. 1 and 2. With the demultiplexed MIMO channel matrix $C(z): \mathbb{C} \longrightarrow \mathbb{C}^{\left(N_{\mathrm{R}} M / 2\right) \times\left(N_{\mathrm{T}} M / 2\right)}$,

$$
\boldsymbol{C}(z)=\left[\begin{array}{ccc}
\boldsymbol{C}_{1,1}(z) & \ldots & \boldsymbol{C}_{1, N_{\mathrm{T}}}(z) \\
\vdots & \ddots & \vdots \\
\boldsymbol{C}_{N_{\mathrm{R}}, 1}(z) & \ldots & \boldsymbol{C}_{N_{\mathrm{R}}, N_{\mathrm{T}}}(z)
\end{array}\right]
$$

which spans the system between $\left[\mathbf{s}_{1}^{\mathrm{H}}[n] \ldots \mathbf{s}_{N_{\mathrm{T}}}^{\mathrm{H}}[n]\right]^{\mathrm{H}}$ and $\left[\mathbf{r}_{1}^{\mathrm{H}}[n] \ldots \mathbf{r}_{N_{\mathrm{R}}}^{\mathrm{H}}[n]\right]^{\mathrm{H}}$ in Fig. 2, we can write

$$
\boldsymbol{F}(z)=\left(\mathbf{I}_{N_{\mathrm{R}}} \otimes \boldsymbol{H}^{\mathrm{P}}(z)\right) \boldsymbol{C}(z)\left(\mathbf{I}_{N_{\mathrm{T}}} \otimes \boldsymbol{H}(z)\right),
$$

where $\otimes$ denotes the Kronecker product.

A few properties of $\boldsymbol{F}(z)$ can be inferred from its structure in (3). Firstly, even though $\boldsymbol{F}(z): \mathbb{C} \rightarrow \mathbb{C}^{\left(N_{\mathrm{R}} M\right) \times\left(N_{\mathrm{T}} M\right)}$, the reduced dimension of $\boldsymbol{C}(z)$ forces the polynomial rank [1] of $\boldsymbol{F}(z)$ to

$$
\operatorname{rank}\{\boldsymbol{F}(z)\} \leq \frac{K M}{2},
$$




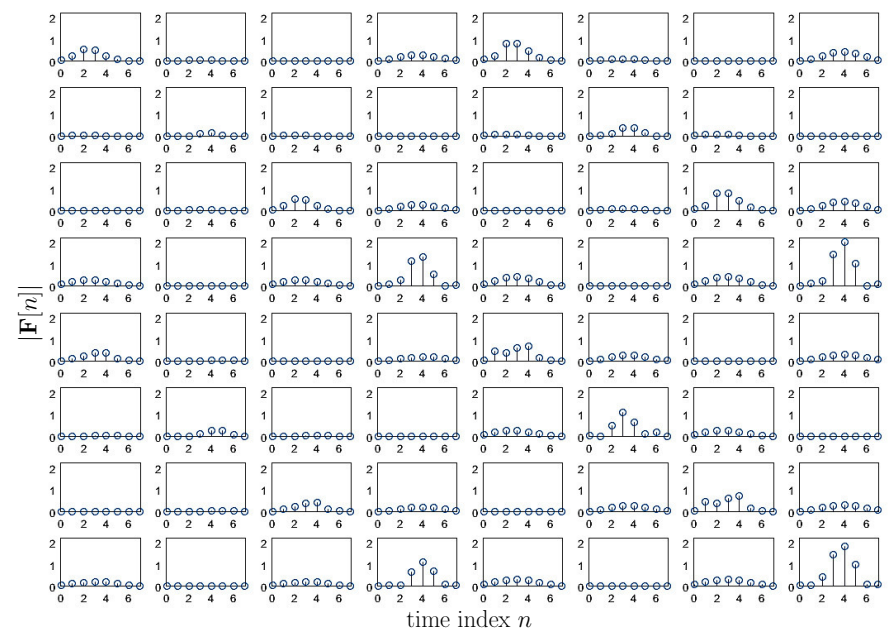

Fig. 3. Example for an equivalent polynomial channel matrix $\boldsymbol{F}(z) \bullet \longrightarrow \mathbf{F}[n]$ for a channel with $N_{\mathrm{T}}=2$ transmitters and $N_{\mathrm{R}}=2$ receivers, and $M=4$ subchannels.

where $K=\min \left\{N_{\mathrm{R}}, N_{\mathrm{T}}\right\}$. Secondly, if the DFT filter bank described by $\boldsymbol{H}(z)$ is constructed for a standard FBMC/OQAM system via [2], then energy will only leak between adjacent bands at most. Therefore, $\boldsymbol{F}(z)$ will be sparse with a block structure, where each $M \times M$ sub-block only contain nonzero elements along its diagonal, its first offdiagonals, and in its upper right and lower left corner elements.

Example. The channel matrix $\mathbf{F}[n]$ for a MIMOFBMC/OQAM system with a $2 \times 2$ random MIMO channel containing impulse responses of length 5 , and $M=4$ subbands is shown in Fig. 3. Therefore, the transfer function $\boldsymbol{F}(z)$ is an $8 \times 8$ sparse polynomial matrix.

\section{MIMO FBMC/OQAM EQUALISATION}

Based on the equivalent channel model derived in Sec. II, we now explore the equalisation of this system. The overall setup in) Fig. 1 contains a subblock $\mathbf{W}[n] \circ-\bullet \boldsymbol{W}(z$ which actions upon the equivalent channel $\mathbf{F}[n] \circ-\bullet \boldsymbol{F}(z)$ prior to OQAM de-staggering of the retrieved $K$ spatial channels of the system. The purpose of this equaliser block is to restore the real orthogonality of the combined system $\boldsymbol{W}(z) \boldsymbol{F}(z)$. Due to the rank-deficiency of $\boldsymbol{F}(z)$, we here have to rely on its pseudo-inverse, which we calculate via the singular value decomposition of a polynomial matrix [8], [9].

While the SVD of a polynomial matrix can also be calculated directly [8], we form two matrices $\boldsymbol{R}_{1}(z): \mathbb{C} \rightarrow$ $\mathbb{C}^{M N_{\mathrm{R}} \times M N_{\mathrm{R}}}$ and $\boldsymbol{R}_{2}(z): \mathbb{C} \rightarrow \mathbb{C}^{M N_{\mathrm{T}} \times M N_{\mathrm{T}}}$ such that

$$
\begin{aligned}
& \boldsymbol{R}_{1}(z)=\boldsymbol{F}(z) \boldsymbol{F}^{\mathrm{P}}(z) \approx \boldsymbol{U}(z) \boldsymbol{\Lambda}_{1}(z) \boldsymbol{U}^{\mathrm{P}}(z) \\
& \boldsymbol{R}_{2}(z)=\boldsymbol{F}^{\mathrm{P}}(z) \boldsymbol{F}(z) \approx \boldsymbol{V}(z) \boldsymbol{\Lambda}_{2}(z) \boldsymbol{V}^{\mathrm{P}}(z) .
\end{aligned}
$$

The factorisations on the r.h.s. of (5) and (6) are polynomials EVDs with paraunitary $\boldsymbol{U}(z)$ and $\boldsymbol{V}(z)$, such that e.g. $\boldsymbol{U}(z) \boldsymbol{U}^{\mathrm{P}}(z)=\boldsymbol{U}^{\mathrm{P}}(z) \boldsymbol{U}(z)=\mathbf{I}$. The approximation sign accounts for the iterative nature of polynomial EVD algorithms in e.g. [4], [9], [16], [17] but also for the fact that the true EVD of the parahermitian matrices $\boldsymbol{R}_{1}(z)$ and $\boldsymbol{R}_{2}$ is almost

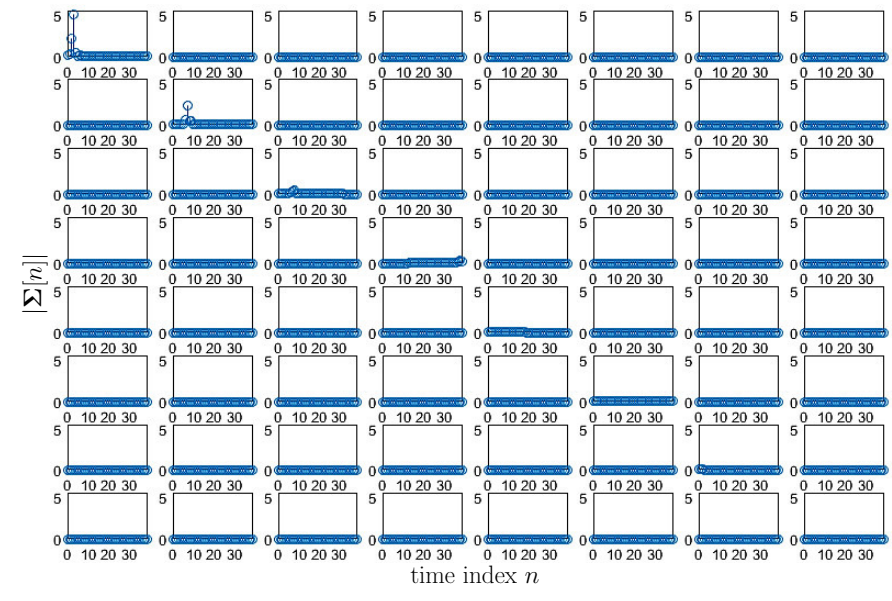

Fig. 4. Matrix $\boldsymbol{\Sigma}[n] \circ-\bullet \boldsymbol{\Sigma}(z)$ of approximate singular values of $\mathbf{F}[n]$ as characterised in Fig. 3.

always infinite in length [21], such that only approximations by polynomials are possible. The terms $\boldsymbol{\Lambda}_{i}(z), i=1,2$ are diagonal parahermitian matrices that approximate the - again likely infinite [21] - eigenvalues of $\boldsymbol{R}_{1}(z)$ and $\boldsymbol{R}_{2}$ by Laurent polynomials.

With the help of (5) and (6) via the evaluation $\boldsymbol{S}(z)=$ $\boldsymbol{U}^{\mathrm{P}}(z) \boldsymbol{F}(z) \boldsymbol{V}(z)$, we can now determine the approximate polynomial SVD

$$
\boldsymbol{F}(z) \approx \boldsymbol{U}(z) \boldsymbol{\Sigma}(z) \boldsymbol{V}^{\mathrm{P}}(z) .
$$

The not necessarily square but diagonal matrix $\Sigma(z): \mathbb{C} \rightarrow$ $\mathbb{C}^{M N_{\mathrm{R}} \times M N_{\mathrm{T}}}$

$$
\boldsymbol{\Sigma}(z)=\operatorname{diag}\left\{\sigma_{1}(z), \sigma_{2}(z), \ldots \sigma_{M K}(z)\right\},
$$

holds the approximate singular values of $\boldsymbol{F}(z)$ [9], extracted from the diagonal of $\boldsymbol{S}(z)$ by neglecting any of its remaining small off-diagonal elements.

The approximate pseudo-inverse of $\boldsymbol{F}(z)$ is now possible via its SVD in (7), such that

$$
\boldsymbol{F}^{\dagger}(z) \approx \boldsymbol{V}(z) \boldsymbol{\Sigma}^{\dagger}(z) \boldsymbol{U}^{\mathrm{P}}(z) .
$$

Pseudo-inverting $\boldsymbol{\Sigma}(z)$ means inverting all singular values $\sigma_{i}(z), i=1 \ldots M K$, as long as these are non-zero, and matrix transposition such that $\boldsymbol{\Sigma}^{\dagger}(z): \mathbb{C} \rightarrow \mathbb{C}^{M N_{\mathrm{T}} \times M N_{\mathrm{R}}}$. Due to the rank deficiency of $\boldsymbol{F}(z)$, at least half of the singular values will be zero, i.e. $\sigma_{i}(z)=0 \forall i=\left(M \frac{K}{2}+1\right) \ldots M K$. The inversion of polynomial terms can be accomplished analytically by partial fraction expansion of $1 / \sigma_{i}(z)$ and approximation of first order terms by geometric series [20], or adaptively using LMS or RLS algorithms [13].

\section{Numerical Example}

We first elaborate on the example of the equivalent polynomial channel matrix $\boldsymbol{F}(z)$ as characterised in Fig. 3. The matrix $\boldsymbol{\Sigma}[n] \circ-\bullet \boldsymbol{\Sigma}(z)$ of approximate singular values is calculated via the multiple-shift SMD algorithm [4], and yields the coefficients shown in Fig. 4. The singular values are 


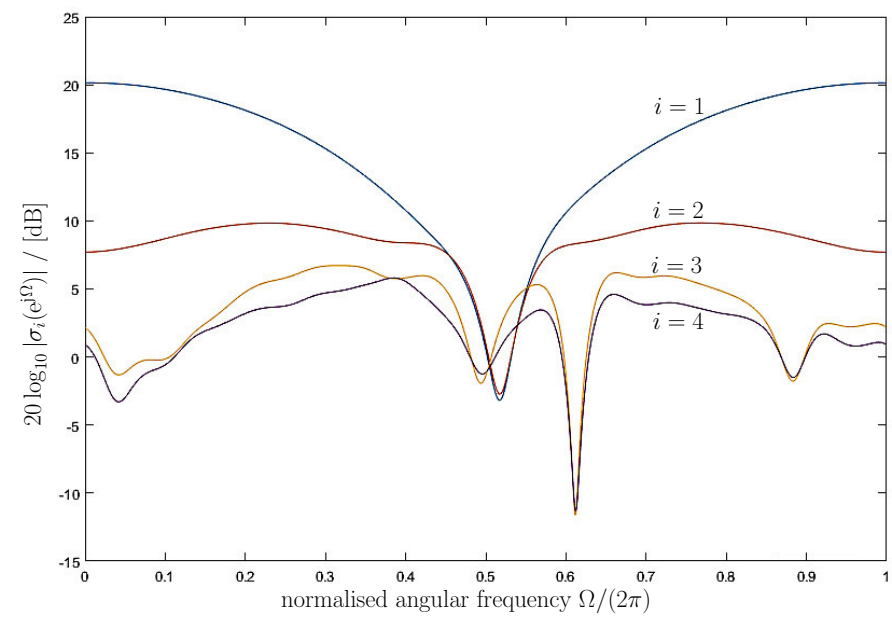

Fig. 5. Evaluation of non-singular values of $\boldsymbol{F}(z)$ in Fig. 3 on the unit circle.

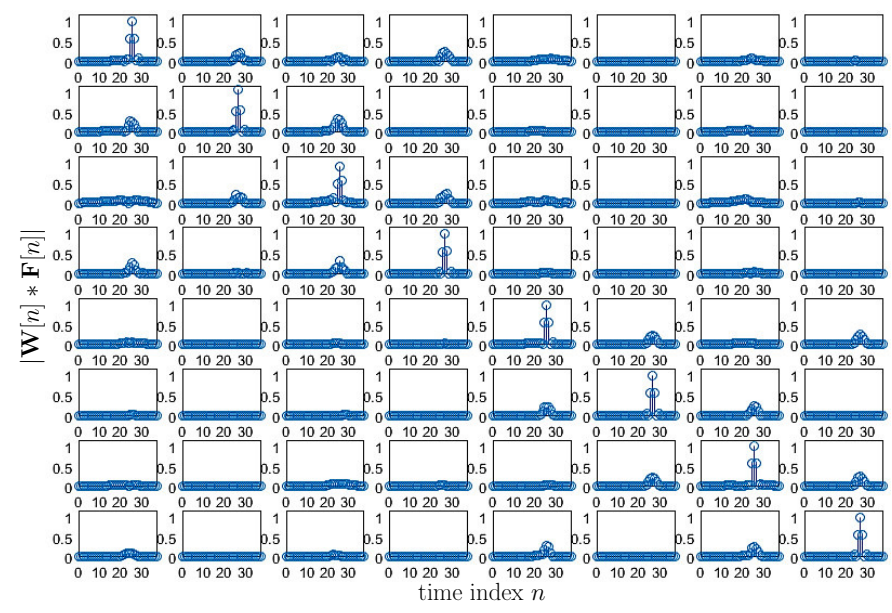

Fig. 6. Overall response of the equivalent channel $\boldsymbol{F}(z) \bullet-\mathbf{F}[n]$, including inner FBMC system of Fig. 2, and the equaliser $\boldsymbol{W}(z) \bullet-0 \mathbf{W}[n]$.

arranged in descending order; moreover, algorithms such as SMD encourage (or in the case of SBR2 can be proven to converge to [10]) spectral majorisation. With $K=2$, the $M K / 2=4$ non-zero singular values can be found in the top left-hand corner of $\boldsymbol{\Sigma}(z)$.

The spectra of the non-zero singular values, i.e. the evaluation of $\sigma_{i}(z)$ on the unit circle for $z=\mathrm{e}^{\mathrm{j} \Omega}$, is shown in Fig. 5, which shows the approximate spectral majorisation, such that

$$
\sigma_{i}\left(\mathrm{e}^{\mathrm{j} \Omega}\right) \gtrsim \sigma_{i+1}\left(\mathrm{e}^{\mathrm{j} \Omega}\right), \quad i=1 \ldots 3, \forall \Omega .
$$

If singular values exhibit strong attenuation or even spectral nulls, their inversion should include a regularisation term, which is particularly advantages in order to avoid noise amplification by the equaliser in the presence of channel noise.

The response of the concatenation of equivalent polynomial channel matrix and equaliser, $\boldsymbol{W}(z) \boldsymbol{F}(z) \bullet-\mathbf{W}[n] * \mathbf{F}[n]$, is shown in Fig. 6. Since $\boldsymbol{F}(z)$ is rank deficient, the system inversion cannot yield an identity matrix. However, sounding the overall MIMO-FBMC/OQAM system of Fig. 1 between

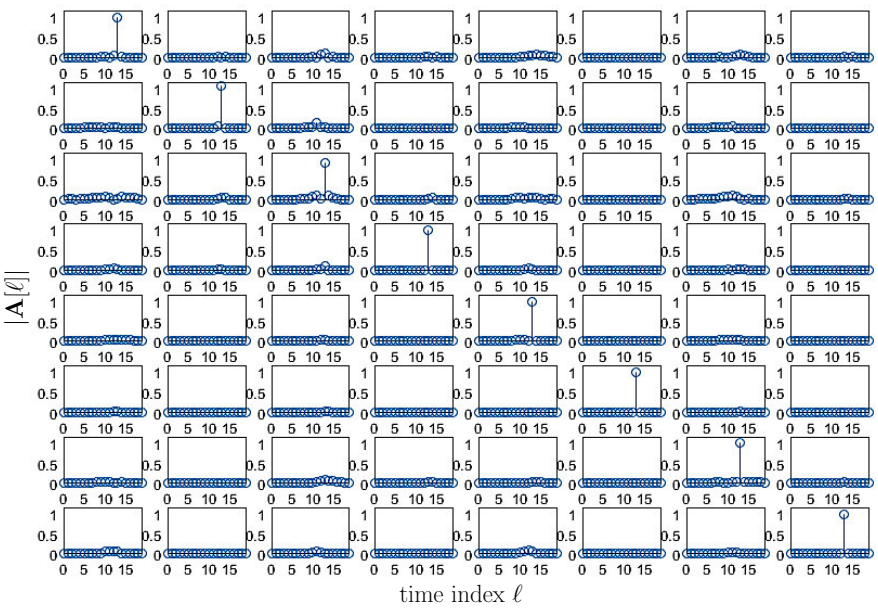

Fig. 7. Response $\mathbf{A}[\ell]$ of the overall MIMO-FBMC/OQAM system as highlighted in Fig. 1.

the inputs $\mathbf{d}_{i}[\ell]$ and outputs $\hat{\mathbf{d}}_{j}[\ell], i, j=1,2$, i.e. we measure the system $\mathbf{A}[\ell]$ such that $\hat{\mathbf{d}}[\ell]=\mathbf{A}[\ell] * \mathbf{d}[\ell]$ with $\mathbf{d}[\ell]=\left[\mathbf{d}_{1}^{\mathrm{H}}[\ell], \mathbf{d}_{2}^{\mathrm{H}}[\ell]\right]^{\mathrm{H}}$ and $\hat{\mathbf{d}}[\ell]=\left[\hat{\mathbf{d}}_{1}^{\mathrm{H}}[\ell], \hat{\mathbf{d}}_{2}^{\mathrm{H}}[\ell]\right]^{\mathrm{H}}$ as shown in Fig. 1, it yield the response depicted in Fig. 7. This response approximates an identity matrix, i.e. inter-symbol, inter-carrier, as well as inter-antenna interference terms have been suppressed by the proposed equaliser.

\section{CONCLUSIONS}

We have investigated the equalisation of an FBMC/OQAM system when extended to a multiple-input multiple-output channel. The equivalent response of the inner MIMOFBMC/OQAM system comprising the filter banks for transmultiplexing as well as the MIMO channel itself, was presented in the form of a polynomial matrix which reflects the different types of distortion (inter-symbol, inter-carrier, and inter-antenna interferences) affecting the system performance. Our proposed equaliser is based on this equivalent channel model characterised by a polynomial matrix. Its structurally imposed rank-deficiency has motivated pseudo-inverse. In a numerical example, we have shown that this system is capable of equalising the overall MIMO-FBMC/OQAM system.

\section{REFERENCES}

[1] V. Belevitch. Classical Network Theory. Holden-Day, 1968.

[2] M. G. Bellanger. Specification and Design of a Prototype Filter for Filter Bank Based Multicarrier transmission. In IEEE International Conference on Acoustics, Speech, and Signal Processing, volume 4, pages 24172420, Salt Lake City, UT, May 2001.

[3] M. Caus and A. Perez-Neira. Multi-stream transmission for highly frequency selective channels in MIMO-FBMC/OQAM systems. IEEE Transactions on Signal Processing, 62(4):786-796, February 2014.

[4] J. Corr, K. Thompson, S. Weiss, I. Proudler, and J. McWhirter. Rowshift corrected truncation of paraunitary matrices for PEVD algorithms. In 23rd European Signal Processing Conference, pages 849-853, Nice, France, August/September 2015.

[5] B. Farhang-Boroujeny. OFDM versus filter bank multicarrier. IEEE Signal Processing Magazine, 28(3):92-112, May 2011.

[6] A. Gilloire and M. Vetterli. Adaptive Filtering in Subbands with Critical Sampling: Analysis, Experiments and Applications to Acoustic Echo Cancelation. IEEE Transactions on Signal Processing, SP-40(8):18621875, August 1992. 
[7] T. Ihalainen, A. Ikhlef, J. Louveaux, and M. Renfors. Channel equalization for multi-antenna fbmc/oqam receivers. IEEE Transactions on Vehicular Technology, 60(5):2070-2085, June 2011.

[8] J. G. McWhirter. An algorithm for polynomial matrix SVD based on generalised Kogbetliantz transformations. In 18th European Signal Processing Conference, pages 457-461, Aalborg, Denmark, August 2010.

[9] J. G. McWhirter, P. D. Baxter, T. Cooper, S. Redif, and J. Foster. An EVD Algorithm for Para-Hermitian Polynomial Matrices. IEEE Transactions on Signal Processing, 55(5):2158-2169, May 2007.

[10] J. G. McWhirter and Z. Wang. A novel insight to the SBR2 algorithm for diagonalising para-hermitian matrices. In 11th IMA Conference on Mathematics in Signal Processing, Birmingham, UK, December 2016.

[11] A. Mertins. MMSE Design of Redundant FIR Precoders for Arbitrary Channel Lengths. IEEE Transactions on Signal Processing, 51(9):24022409, September 2003.

[12] X. Mestre and D. Gregoratti. A parallel processing approach to filterbank multicarrier MIMO transmission under strong frequency selectivity. In IEEE International Conference on Acoustics, Speech and Signal Processing, pages 8078-8082, May 2014.

[13] A. Nagy and S. Weiss. Synchronisation and equalisation of an fbmc/oqam system by a polynomial matrix pseudo-inverse. In IEEE International Symposium on Signal Processing and Information Technology, Bilbao, December 2017.

[14] R. Nissel, S. Schwarz, and M. Rupp. Filter bank multicarrier modulation schemes for future mobile communications. IEEE Journal on Selected Areas in Communications, 35(8):1768-1782, August 2017.

[15] A. I. Prez-Neira, M. Caus, R. Zakaria, D. L. Ruyet, E. Kofidis, M. Haardt, X. Mestre, and Y. Cheng. MIMO signal processing in offsetQAM based filter bank multicarrier systems. IEEE Transactions on Signal Processing, 64(21):5733-5762, November 2016.

[16] S. Redif, J. McWhirter, and S. Weiss. Design of FIR paraunitary filter banks for subband coding using a polynomial eigenvalue decomposition. IEEE Transactions on Signal Processing, 59(11):5253-5264, November 2011.

[17] S. Redif, S. Weiss, and J. McWhirter. Sequential matrix diagonalization algorithms for polynomial EVD of parahermitian matrices. IEEE Transactions on Signal Processing, 63(1):81-89, January 2015.

[18] P. Siohan, C. Siclet, and N. Lacaille. Analysis and Design of OFDM/OQAM Systems Based on Filterbank Theory. IEEE Transactions on Signal Processing, 50(5):1170-1183, May 2002.

[19] P. P. Vaidyanathan. Multirate Systems and Filter Banks. Prentice Hall, Englewood Cliffs, 1993.

[20] S. Weiss, A. Millar, and R. W. Stewart. Inversion of parahermitian matrices. In 18th European Signal Processing Conference, pages 447451, Aalborg, Denmark, August 2010.

[21] S. Weiss, J. Pestana, and I. Proudler. On the existence and uniqueness of the eigenvalue decomposition of a parahermitian matrix. IEEE Transactions on Signal Processing, 2018. to appear. 